\title{
The effects of probiotic and conventional yoghurt on lipid profile in women
}

\author{
Haleh Sadrzadeh-Yeganeh ${ }^{1}$, Ibrahim Elmadfa ${ }^{2} *$ Abolghasem Djazayery $^{1}$, Mahmoud Jalali ${ }^{1}$, \\ Ramin Heshmat ${ }^{3}$ and Maryam Chamary ${ }^{1}$ \\ ${ }^{1}$ Department of Nutritional Sciences, School of Public Health, Tehran University of Medical Sciences, Tehran, Iran \\ ${ }^{2}$ Department of Nutritional Sciences, University of Vienna, Austria \\ ${ }^{3}$ Endocrinology and Metabolism Research Center, Tehran University of Medical Sciences, Tehran, Iran
}

(Received 12 August 2009 - Revised 10 December 2009 - Accepted 11 December 2009 - First published online 26 January 2010)

Many studies have been done on the hypocholesterolaemic effect of probiotic yoghurt. The results, however, are not conclusive. The aim of the present study was to test the effect of probiotic and conventional yoghurt on the lipid profile in women. In a randomised trial, ninety female volunteers aged 19-49 years were assigned to three groups. Subjects consumed daily 300 g probiotic yoghurt containing Lactobacillus acidophilus La5 and Bifidobacterium lactis Bb12 or $300 \mathrm{~g}$ conventional yoghurt or no yoghurt for 6 weeks. Fasting blood samples, $3 \mathrm{~d}$ dietary records and anthropometric measurements were collected at baseline (T1), end of week 3 (T2) and end of week 6 (T3). Lipid profile parameters were determined by enzymic methods. Results showed no significant difference in lipid profile within any group throughout the study. Comparing mean differences $(\mathrm{T} 1-\mathrm{T} 3)$ among the three groups showed: no difference in TAG and LDL-cholesterol, a decrease in cholesterol in both conventional $(P<0.05)$ and probiotic yoghurt groups $(P<0.005)$ compared with the control group, a decrease in total:HDL-cholesterol ratio for conventional $(P<0.05)$ and probiotic yoghurt groups $(P<0.001)$ compared with the control group, and an increase in HDL-cholesterol in the probiotic yoghurt group $(P<0.05)$ compared with the control group. Positive changes in lipid profile were observed in both yoghurt groups. Any added effect, therefore, is due to the consumption of fermented milk products.

Probiotic yoghurt: Lipid profile: Women

CVD is considered as a multifactorial disease which has a high mortality rate. Abnormal levels of blood lipids are among the risk factors for $\mathrm{CVD}^{(1,2)}$. The cholesterol-lowering effect of probiotics has been the centre of attention in numerous studies. The term probiotics denotes 'for life' and refers to micro-organisms which have positive effects on human health. Micro-organisms presiding in food, especially lactic acid bacteria, were believed to have beneficial health advantages from long ago. As probiotics, certain species among bifidobacteria and lactobacilli can help balance the intestine's microflora. This balance is formed by increasing the number of helpful bacteria and reducing pathogenic bacteria in the intestine. Factors such as inadequate nutrition, antibiotics, stress, ageing and infections can disturb this balance. Whenever this state of equilibrium is upset within the intestine, the harmful bacteria find a chance to thrive and multiply. The growth of these bacteria causes digestive ailments and illnesses ${ }^{(3)}$. In addition, balanced microflora can improve the barrier function of the gut, influence protective functions of the intestinal mucosa and modify its immune response ${ }^{(4-6)}$. Releasing antimicrobial substances in the body, resisting colonisation of harmful bacteria, stopping pathogens from attaching to the gastrointestinal tract, breaking up destructive elements and boosting the activity of brushborder enzymes are among the actions of probiotics.

In their study of the Maasai tribes, Mann \& Spoerry noted for the first time that consumption of fermented milk reduced serum cholesterol concentration ${ }^{(7)}$. In vitro experiments with some strains of bifidobacteria and lactobacilli reported that they were able to assimilate cholesterol if bile acids were present ${ }^{(8-11)}$.

Probiotic bacteria can ferment indigestible carbohydrates in the food and produce SCFA in the gut. This can cause a reduction in the systemic levels of blood lipids ${ }^{(12)}$. Some of these bacteria can assimilate cholesterol directly from the gastrointestinal tract or deconjugate bile salts and hence have an effect on the process of cholesterol absorption ${ }^{(8,10)}$. Some studies have attributed the hypocholesterolaemic effects of fermented milk to the sphingolipids present in yoghurt and the fatty acids in milk fat ${ }^{(13,14)}$.

Sphingolipids can be found in lipid-rich structures, such as cellular membranes, and especially lipoproteins. They have an effect on the process of cholesterol metabolism and transport.

Even though some human and animal studies have suggested a moderate cholesterol-lowering property of some fermented dairy products, clinical studies on human subjects

Abbreviations: T1, data collection at the beginning of the study; T2, data collection after 3 weeks; T3, data collection after 6 weeks; TUMS, Tehran University of Medical Sciences.

*Corresponding author: Dr Ibrahim Elmadfa, fax +4314277 9549, email ibrahim.elmadfa@univie.ac.at 
have not been conclusive ${ }^{(13-20)}$. The contradictory outcome is most probably due to different experimental designs, different strains of lactic bacteria, and intakes of fermented milk as a confounding factor, thereby leading to difficulty in evaluating the results.

The evidence about the effect of probiotic yoghurt on lipid profile has shown conflicting results. Furthermore, in the studies that were conducted, usually probiotic and conventional yoghurt-consuming groups were compared. In the present study, in addition to the two yoghurt groups, a third group which did not use fermented milk was considered as a control group. The aim of the present study was to investigate the effect of probiotic yoghurt containing Lactobacillus acidophilus La5 and Bifidobacterium lactis $\mathrm{Bb} 12$ and conventional yoghurt on the lipid profile in healthy women.

\section{Subjects and methods}

The present study was a randomised clinical trial. A total of ninety female volunteers aged between 19 and 49 years, with cholesterol levels less than $6.2 \mathrm{mmol} / \mathrm{l}$, TAG levels less than $2.3 \mathrm{mmol} / \mathrm{l}$ and a BMI less than $30 \mathrm{~kg} / \mathrm{m}^{2}$ were recruited from the personnel and students of Tehran University of Medical Sciences (TUMS) through an advertisement. Exclusion criteria were: smoking; having kidney, liver or inflammatory intestinal disease, thyroid, diabetes, disorders, immunodeficiency diseases, lactose intolerance; taking supplements, taking medication; consuming probiotic yoghurt or any other probioticcontaining products in the last 2 months; being elite athletes, pregnant or breast-feeding.

A 1-week pre-adjustment period was designated, during which all subjects had to refrain from taking yoghurt or any other fermented food. Subjects were randomly assigned into three groups, each group consisting of thirty individuals. The first and second groups consumed $300 \mathrm{~g}$ daily of probiotic and conventional yoghurt, respectively. The third group, as the control group, did not consume any fermented and probiotic products for the duration of the study (6 weeks). The volunteers were told not to alter their exercise routine or regular diet, and not to consume any yoghurt other than the one provided to them by the researcher. They were also asked to refrain from consuming any other probiotic and fermented products. The study was triple blind for the yoghurt-consuming groups. That is, in addition to the subjects and the investigator, the evaluator of the results was also not aware which treatment any particular subject received. Necessary arrangements were made so that every week the subjects would receive a week's supply of their probiotic or conventional yoghurts directly from the factory. Of the subjects, two had to be excluded from the study at week 1 because of taking antibiotics: one from the probiotic group and one from the control group.

Both the probiotic and conventional yoghurts that were supplied contained Lactobacillus bulgaricus and Streptococcus thermophilus. The probiotic yoghurt was also enriched with adding cultures of B. lactis $\mathrm{Bb} 12$ and L. acidophilus La5 (Chr Hansen, Denmark). Direct Vat Set (DVS) cultures were used. Microbiological analysis of the probiotic yoghurt showed that it contained $3.9 \times 10^{7}$ colony-forming units (cfu) of both B. lactis Bb12 and L. acidophilus La5. The analysis on conventional yoghurt confirmed the presence of $10^{6}-10^{7} \mathrm{cfu}$ of S. thermophilus and L. bulgaricus. The yoghurts' acidity was $\mathrm{pH} 4.3$. The fat content was $2.5 \%$, comparable in both yoghurt types.

\section{Data collection}

Information on food consumption (through $3 \mathrm{~d}$ dietary records), fasting blood samples and anthropometric measurements were collected at three intervals: at the beginning of the trial (T1), at the end of week 3 (T2) and at the end of week 6 (T3). Compliance with the yoghurt consumption guidelines at home was monitored once per week through phone interviews.

At each of the three intervals, body weights were measured (digital floor scale; Seca, Hamburg, Germany) with $0.1 \mathrm{~kg}$ accuracy without shoes and with minimum clothing. Weight was measured in the fasting state. The subjects' heights were measured, with $0.1 \mathrm{~cm}$ accuracy, with non-stretchable tape (Seca). BMI was determined by dividing body weight by height squared $\left(\mathrm{kg} / \mathrm{m}^{2}\right)$.

At the end of each interval $3 \mathrm{~d}$ dietary records were taken from each volunteer, who was instructed to write down the types and amounts of foods eaten. The dietary record comprised of one weekend and two non-consecutive weekdays. The amount of food eaten by each subject was estimated from household measures and from photographs of portion sizes ${ }^{(21)}$. Through weekly follow-ups by phone, and through periodical visits of the subject at each interval to the Nutrition Department at TUMS, a nutritionist checked the subjects' compliance with the study protocol and assessed dietary records in person.

The subjects were directed to report at TUMS at the end of each interval for blood tests. The blood sample was drawn from the antecubital vein in the arm. The blood was drawn after a $14 \mathrm{~h}$ fast, in the morning. Serum total cholesterol and TAG concentrations were measured enzymically with Parsazmun's kits (DiaSys, Germany) using cholesterol oxidase$p$-aminophenazone (CHOD-PAP) and glycerol phosphate oxidase- $p$-aminophenazon (GPO-PAP) ${ }^{(22,23)}$. HDL-cholesterol and LDL-cholesterol were also measured enzymically with Parsazmun's kits (DiaSys, Germany) ${ }^{(24)}$.

\section{Statistical analysis}

The data were analysed using ANOVA. ANOVA was used to identify any differences among the three groups or among the three intervals within a group. The Kolmogorov-Smirnov test was used to test the normality of the distribution of variables. For distributions that were not normal the non-parametric test of Kruskal-Wallis was used to compare the groups, and the data were expressed in medians and interquartile ranges of the original variable. Otherwise, the data were expressed as means and standard deviations. Multiple comparisons were conducted by the Bonferroni post hoc test. The statistical tests were conducted using SPSS (version 11.5; SPSS, Inc., Chicago, IL, USA). Food Processor II (ESHA, Salem, OR, USA) was used in performing nutrient calculations for the $3 \mathrm{~d}$ dietary records. The database of this software is built upon the Nutrient Database Bank for Standard Reference from the US Department of Agriculture and other sources. The database was modified with reference to the existing 
national Iranian food composition table, developed by the Iranian National Institute of Nutrition and Food Technology. In developing the regional food composition database, biological variation, fortification and enrichment of food items (such as rice, wheat, dairy products, oils, etc) were taken into account. This program is used for analysing national nutrition surveys in Iran. $P<0.05$ was considered as statistically significant.

The present study was conducted according to the guidelines laid down in the Declaration of Helsinki and all procedures involving human subjects were approved by the Ethics Committee at TUMS. Written informed consent was obtained from all subjects.

\section{Results}

The mean values and standard deviations of weight, height, age and BMI of the volunteers at the beginning of the study did not indicate any significant differences among the three study groups. The mean age of the conventional and probiotic groups was 32 and 35.5 years, respectively, and 34.7 years for the control group.

ANOVA did not show any significant change in weight and BMI of the subjects at the three intervals of the study within a group (Table 1). ANOVA did not show any difference among the three groups at the beginning of the study for intakes of total fat, SFA, MUFA, PUFA and dietary fibre, based on the $3 \mathrm{~d}$ dietary records. There was no significant change in the intakes of these parameters during the study, within any group (Table 1). The Kruskal-Wallis test showed no statistically significant change in total energy and cholesterol intakes at the beginning of the study among the three groups. There was no significant difference in total energy or cholesterol intake throughout the study within a group (Table 1).

ANOVA showed that there was no statistically significant difference among the three groups in blood lipid parameters at baseline. Neither was there any statistically significant difference throughout the study for blood lipid parameters within any group (Table 2). In the probiotic yoghurt group, a descending trend in total:HDL-cholesterol ratio of $12.5 \%$ $(P>0.05)$ was observed.

Comparing the mean differences between baseline (T1) and 6 weeks (T3) for TAG and LDL-cholesterol showed no statistically significant difference among the three groups (Table 3). The post hoc test showed that the decrease (T1 - T3) in total cholesterol was significant for both the conventional and probiotic yoghurt groups $(P=0.014$ and $P=0.002$, respectively) compared with the control group. The increase in the mean difference (T1 - T3) concentration of HDL-cholesterol was significant only for probiotic yoghurt compared with the control group $(P=0.009)$. For the total:HDL-cholesterol ratio the post hoc test showed that the decrease was significant for both the conventional and probiotic yoghurt groups compared with the control group ( $P=0.006$ and $P=0.000$, respectively) (Table 3 ).

Table 1. Subjects' weight, BMI and nutrient intakes (3d dietary records) throughout the study

(Mean values and standard deviations)

\begin{tabular}{|c|c|c|c|c|c|c|c|c|}
\hline \multirow[b]{2}{*}{ Variables } & \multirow[b]{2}{*}{ Groups } & \multicolumn{2}{|c|}{ T1 } & \multicolumn{2}{|c|}{ T2 } & \multicolumn{2}{|c|}{ T3 } & \multirow[b]{2}{*}{$P$} \\
\hline & & Mean & SD & Mean & SD & Mean & SD & \\
\hline \multirow[t]{3}{*}{ Weight (kg) } & Conventional yoghurt & 58.5 & $6 \cdot 8$ & $58 \cdot 4$ & $7 \cdot 0$ & $58 \cdot 9$ & $7 \cdot 1$ & 0.958 \\
\hline & Probiotic yoghurt & $60 \cdot 7$ & $7 \cdot 0$ & $60 \cdot 8$ & $7 \cdot 1$ & $60 \cdot 9$ & $7 \cdot 4$ & 0.994 \\
\hline & Control & $59 \cdot 3$ & $7 \cdot 3$ & $59 \cdot 3$ & $7 \cdot 2$ & $59 \cdot 3$ & $7 \cdot 3$ & 1.000 \\
\hline \multirow[t]{3}{*}{ BMI $\left(\mathrm{kg} / \mathrm{m}^{2}\right)$} & Conventional yoghurt & $23 \cdot 0$ & 2.4 & 23.0 & 2.6 & $23 \cdot 2$ & 2.6 & 0.954 \\
\hline & Probiotic yoghurt & $24 \cdot 0$ & $2 \cdot 4$ & $24 \cdot 1$ & 2.5 & $24 \cdot 0$ & $2 \cdot 6$ & 0.985 \\
\hline & Control & $23 \cdot 8$ & 3.0 & 23.7 & 2.9 & $23 \cdot 8$ & $2 \cdot 8$ & 0.999 \\
\hline \multirow[t]{3}{*}{ Total fat (\% energy) } & Conventional yoghurt & $34 \cdot 0$ & $5 \cdot 0$ & 34.7 & $6 \cdot 9$ & 33.9 & $5 \cdot 3$ & 0.859 \\
\hline & Probiotic yoghurt & $35 \cdot 7$ & 5.4 & 33.5 & $7 \cdot 1$ & $33 \cdot 8$ & 5.9 & 0.342 \\
\hline & Control & $35 \cdot 7$ & 5.6 & $35 \cdot 0$ & $5 \cdot 6$ & $35 \cdot 1$ & $6 \cdot 3$ & 0.878 \\
\hline \multirow[t]{3}{*}{ SFA (\% energy) } & Conventional yoghurt & $11 \cdot 0$ & $2 \cdot 4$ & $11 \cdot 1$ & $2 \cdot 7$ & $11 \cdot 3$ & $2 \cdot 2$ & 0.841 \\
\hline & Probiotic yoghurt & $10 \cdot 9$ & $2 \cdot 3$ & 11.9 & $2 \cdot 6$ & 11.5 & $2 \cdot 0$ & 0.292 \\
\hline & Control & 11.5 & 2.9 & $10 \cdot 6$ & 1.6 & $10 \cdot 9$ & 1.9 & 0.283 \\
\hline \multirow[t]{3}{*}{ MUFA (\% energy) } & Conventional yoghurt & $10 \cdot 1$ & 1.6 & 10.5 & 2.5 & $10 \cdot 7$ & $1 \cdot 8$ & 0.582 \\
\hline & Probiotic yoghurt & $11 \cdot 0$ & $2 \cdot 3$ & $10 \cdot 1$ & $2 \cdot 0$ & $10 \cdot 2$ & 1.9 & 0.199 \\
\hline & Control & $10 \cdot 7$ & 1.7 & 10.5 & $2 \cdot 2$ & $10 \cdot 8$ & 2.4 & 0.836 \\
\hline \multirow[t]{3}{*}{ PUFA (\% energy) } & Conventional yoghurt & 11.5 & 3.7 & $11 \cdot 0$ & $3 \cdot 2$ & $10 \cdot 4$ & $3 \cdot 6$ & 0.487 \\
\hline & Probiotic yoghurt & 11.4 & $3 \cdot 1$ & 9.5 & 3.9 & $9 \cdot 9$ & $3 \cdot 6$ & 0.091 \\
\hline & Control & $11 \cdot 1$ & 3.6 & 11.6 & $3 \cdot 8$ & $11 \cdot 1$ & $4 \cdot 2$ & 0.831 \\
\hline \multirow[t]{3}{*}{ Dietary fibre (g) } & Conventional yoghurt & $16 \cdot 5$ & 5.4 & 17.9 & 6.5 & $18 \cdot 3$ & $8 \cdot 7$ & 0.590 \\
\hline & Probiotic yoghurt & $16 \cdot 7$ & $6 \cdot 7$ & $16 \cdot 5$ & $7 \cdot 0$ & $14 \cdot 8$ & $5 \cdot 4$ & 0.458 \\
\hline & Control & $16 \cdot 8$ & $7 \cdot 1$ & $17 \cdot 1$ & $7 \cdot 2$ & $18 \cdot 0$ & 8.4 & 0.848 \\
\hline \multirow[t]{3}{*}{ Total energy $(\mathrm{kJ})^{\star}$} & Conventional yoghurt & 6893.6 & $2427 \cdot 1$ & $7488 \cdot 1$ & $2385 \cdot 2$ & 7249.4 & 3289.6 & 0.392 \\
\hline & Probiotic yoghurt & 7058.9 & $2376 \cdot 0$ & $6849 \cdot 6$ & $1605 \cdot 6$ & 7213.9 & $2652 \cdot 3$ & 0.926 \\
\hline & Control & 6937.5 & 2323.7 & $6887 \cdot 3$ & $3112 \cdot 9$ & $6791 \cdot 0$ & $3112 \cdot 9$ & 0.873 \\
\hline \multirow[t]{3}{*}{ Cholesterol $(\mathrm{mg})^{\star}$} & Conventional yoghurt & $162 \cdot 5$ & $139 \cdot 8$ & 206.5 & $161 \cdot 7$ & $195 \cdot 0$ & $222 \cdot 0$ & 0.608 \\
\hline & Probiotic yoghurt & $146 \cdot 0$ & 138.0 & $199 \cdot 0$ & 134.5 & $158 \cdot 0$ & $170 \cdot 8$ & 0.380 \\
\hline & Control & $142 \cdot 0$ & 136.5 & $169 \cdot 0$ & $106 \cdot 0$ & 173.0 & 173.5 & 0.518 \\
\hline
\end{tabular}

T1, data collection at the beginning of the study; T2, data collection after 3 weeks; T3, data collection after 6 weeks.

${ }^{\star}$ Median and interquartile ranges. 
Table 2. Blood lipid parameters of the subjects during the study (Mean values and standard deviations)

\begin{tabular}{|c|c|c|c|c|c|c|c|c|}
\hline \multirow[b]{2}{*}{ Variables (mmol/l) } & \multirow[b]{2}{*}{ Groups } & \multicolumn{2}{|c|}{$\mathrm{T} 1$} & \multicolumn{2}{|c|}{ T2 } & \multicolumn{2}{|c|}{ T3 } & \multirow[b]{2}{*}{$P$} \\
\hline & & Mean & SD & Mean & SD & Mean & SD & \\
\hline \multirow[t]{3}{*}{ Total cholesterol } & Conventional yoghurt & 4.50 & 0.66 & 4.49 & 0.75 & 4.38 & 0.58 & 0.727 \\
\hline & Probiotic yoghurt & 4.81 & 0.62 & 4.78 & 0.72 & 4.61 & 0.66 & 0.493 \\
\hline & Control & 4.78 & 0.72 & 4.79 & 0.88 & 4.96 & 0.94 & 0.671 \\
\hline \multirow[t]{3}{*}{ TAG } & Conventional yoghurt & 0.89 & 0.25 & 0.93 & 0.22 & 0.92 & 0.24 & 0.828 \\
\hline & Probiotic yoghurt & $1 \cdot 11$ & 0.45 & $1 \cdot 14$ & 0.43 & $1 \cdot 11$ & 0.41 & 0.972 \\
\hline & Control & 1.08 & 0.44 & 1.14 & 0.36 & $1 \cdot 16$ & 0.38 & 0.752 \\
\hline \multirow[t]{3}{*}{ HDL-cholesterol } & Conventional yoghurt & 1.31 & 0.23 & 1.41 & 0.21 & $1 \cdot 38$ & 0.23 & 0.241 \\
\hline & Probiotic yoghurt & 1.27 & 0.26 & 1.37 & 0.21 & 1.39 & 0.24 & 0.153 \\
\hline & Control & 1.27 & 0.31 & 1.31 & 0.35 & 1.26 & 0.32 & 0.839 \\
\hline \multirow[t]{3}{*}{ LDL-cholesterol } & Conventional yoghurt & $2 \cdot 62$ & 0.50 & $2 \cdot 67$ & 0.57 & 2.59 & 0.50 & 0.825 \\
\hline & Probiotic yoghurt & 2.85 & 0.46 & 2.85 & 0.54 & $2 \cdot 79$ & 0.54 & 0.885 \\
\hline & Control & 2.85 & 0.60 & 2.94 & 0.63 & 2.93 & 0.63 & 0.830 \\
\hline \multirow[t]{3}{*}{ Total:HDL cholesterol ratio } & Conventional yoghurt & 0.09 & 0.02 & 0.08 & 0.02 & 0.08 & 0.02 & 0.183 \\
\hline & Probiotic yoghurt & 0.10 & 0.02 & 0.09 & 0.02 & 0.09 & 0.02 & 0.058 \\
\hline & Control & 0.10 & 0.03 & 0.10 & 0.04 & 0.11 & 0.04 & 0.592 \\
\hline
\end{tabular}

T1, blood sample collection at the beginning of the study; T2, blood sample collection after 3 weeks; T3, blood sample collection after 6 weeks.

\section{Discussion}

In the present study there were no statistically significant differences between the three groups in terms of weight and BMI. It is possible that by introducing yoghurt to the diet of subjects, a change in energy intake and, consequently, weight gain occurs. We did not find any significant change in weight, and BMI within any group during the study.

Though we did not observe statistically significant changes in nutrient intakes during the course of the study, there was a descending trend in the intake of total energy and SFA in the control group, compared with the two yoghurt-consuming groups (Table 1). The reason for the observed trend could be that consumption of yoghurt as a side-dish is part of a dietary habit in Iran and yoghurt is one of the main items in the daily household basket. For the two yoghurt-consuming groups, their dietary yoghurt was substituted by the experimental products. But for the control group, yoghurt consumption was restricted and it was withdrawn from their diet. Although an equal amount of energy, through other foods, was introduced to the diet of the control group to compensate for the yoghurt restriction, in terms of fat content, it could have resulted in a reduction in energy and saturated fat intake in the control group.

The present results showed no statistically significant difference in lipid profile parameters within any group throughout the study. There was a lowering effect $(\mathrm{T} 1-\mathrm{T} 3$ mean difference) due to the consumption of both conventional and probiotic yoghurts on total cholesterol and total:HDL-cholesterol ratio compared with the control group. We did not observe any significant differences in lipid profile between the two yoghurt groups. We did, however, observe significant differences when compared with the control group. At baseline, the PUFA:SFA ratio was 1.03 for both the conventional and probiotic yoghurt groups. This ratio was 0.95 for the control group. In the control group, throughout the study the ratio increased due to a decrease in SFA and the mean value of PUFA remained almost constant at $\mathrm{T} 1$ and $\mathrm{T} 3$ intervals. The increase observed in plasma total cholesterol and total:HDL-cholesterol ratio in the control group could be due to the increase in the intake of cholesterol in this group. In the yoghurt-consuming groups, too, an increase in the intake of cholesterol was observed, but there was a decrease in PUFA:SFA ratios. Despite these changes, there was an improvement in plasma total cholesterol and total:HDLcholesterol ratio in the yoghurt-consuming groups. Consumption of fermented milk could account for the reduction in cholesterol concentration in the present study. Some early studies on fermented milk also showed cholesterol-lowering effects of these products ${ }^{(25)}$. Adding $L$. acidophilus to infant formula resulted in a lower serum cholesterol concentration compared with the control formula ${ }^{(26)}$.

Table 3. Comparing differences between baseline (T1) mean and week 6 (T3) mean of blood lipid parameters for each group (Mean values and standard deviations and percentage changes)

\begin{tabular}{|c|c|c|c|c|c|c|c|c|c|c|}
\hline \multirow[b]{2}{*}{ Variables (mmol/l) } & \multicolumn{3}{|c|}{ Conventional yoghurt ( $n$ 30) } & \multicolumn{3}{|c|}{ Probiotic yoghurt ( $n$ 29) } & \multicolumn{3}{|c|}{ Control ( $n$ 29) } & \multirow[b]{2}{*}{$P$} \\
\hline & Mean & SD & Change (\%) & Mean & SD & Change (\%) & Mean & SD & Change (\%) & \\
\hline Total cholesterol & $-0.13^{\star}$ & 0.36 & $-2 \cdot 8$ & $-0.20^{\star \star}$ & 0.33 & $-4 \cdot 1$ & 0.18 & 0.51 & 3.8 & 0.001 \\
\hline TAG & 0.03 & $0 \cdot 13$ & 3.6 & -0.00 & 0.30 & -0.2 & 0.08 & 0.26 & $7 \cdot 1$ & 0.423 \\
\hline HDL-cholesterol & 0.07 & 0.17 & $5 \cdot 2$ & $0.11^{\star *}$ & 0.15 & 8.8 & -0.01 & 0.13 & -0.8 & 0.011 \\
\hline LDL-cholesterol & -0.03 & 0.24 & $-1 \cdot 1$ & -0.06 & 0.29 & $-2 \cdot 0$ & 0.08 & 0.43 & $3 \cdot 0$ & 0.243 \\
\hline Total:HDL-cholesterol ratio & $-0.01^{\star *}$ & 0.01 & -7.7 & $-0.01^{\star *}$ & 0.01 & $-12 \cdot 6$ & 0.01 & 0.02 & $6 \cdot 1$ & 0.000 \\
\hline
\end{tabular}

Mean value was significantly different from that of the control group: ${ }^{*} P<0.05,{ }^{* *} P<0.01$. 
In a study by Schaafsma et al. a significant reduction of serum cholesterol concentrations was seen with daily consumption of fermented milk containing two strains of L. acidophilus after $21 \mathrm{~d}^{(27)}$. The yoghurt also had $2.5 \%$ fructo-oligosaccharides, which may have affected serum cholesterol concentrations. In a study by Agerbaek et al. on the effect of a milk product, fermented with Enterococcum faecium and two strains of $S$. thermophilus, on lipid profile, a significant reduction in LDL-cholesterol concentration was found after 6 weeks of daily consumption of a fermented milk product compared with a placebo (milk acidified with an organic acid) ${ }^{(28)}$. Long-term consumption of the same product resulted in LDL-cholesterol reduction similar to the placebo group, indicating that both low-fat milk and fermented milk products may have some hypocholesterolaemic effects ${ }^{(29)}$.

The significant decrease in the mean difference of total:HDL-cholesterol ratio in both yoghurt groups compared with the control in the present study could be the consequence of decreases in the total cholesterol level as well as increases in HDL-cholesterol concentration after consuming fermented milk for 6 weeks ${ }^{(14,27)}$.

The observed effect in both yoghurt groups could be because of sphingolipids in yoghurt and the distribution of milk fat ${ }^{(13,14)}$. Short- and long-term animal studies with rats have shown that sphingolipids reduce plasma cholesterol ${ }^{(30,31)}$. Some studies have shown that sphingolipids have an LDLcholesterol- and total cholesterol-reducing effect and an increasing effect on HDL-cholesterol ${ }^{(32)}$. Other studies have indicated that sphingolipids have a reversing effect on the LDL-:HDL-cholesterol ratio ${ }^{(33)}$. Other studies suggested that the increase in HDL-cholesterol and decrease in total:HDLcholesterol ratio is because of SFA (12:0 and 14:0) in milk $\mathrm{fat}^{(34-36)}$. The conventional and probiotic yoghurts that we used in the present study contained $2.5 \%$ fat. Therefore, sphingolipid contents in both yoghurts should be similar. It should be noted, however, that another potential source of sphingolipids is cell membranes of bacteria themselves, which were higher in the probiotic yoghurt. This could have led to a higher sphingolipids content in the probiotic yoghurt and as a result an increase in HDL-cholesterol.

Furthermore, the results from some studies that used probiotic capsules instead of dairy products as a vehicle for administrating probiotics did not support the cholesterollowering effect of probiotic bacteria ${ }^{(20,37)}$. Rather, they supported the claim that a dairy product is a more effective medium of administering probiotic bacteria. It was proposed that orotic acid and 3-hydroxyl-methyl glutarate in milk, independently, could have cholesterol-lowering effects.

In the present study, other mechanisms could be responsible for the hypocholesterolaemic effect that we observed in the group that consumed probiotic yoghurt. It has been proposed that when probiotics settle in the gut, they ferment indigestible carbohydrate from food. Their action raises the SCFA in the gut. SCFA are produced from peptides, polysaccharides, proteins and oligosaccharides, mainly by anaerobic bacteria, and are the final product of bacterial activity in the gastrointestinal tract. These large molecules are depolymerised by a variety of hydrolytic enzymes that are produced by bacteria and allow the organisms to ferment their sugar content. SCFA can lower the lipids in blood through blocking the synthesis of hepatic cholesterol and/or through redirecting plasma cholesterol toward the liver. By producing bile acids through deconjugating the bile salts in the small intestine, probiotics prevent micelle production. When cholesterol enters the enterohepatic circulation, it is dealt with in the same way. Probiotics, by using hydroxy steroid dehydrogenase and conjugated bile acid hydrolase enzymes, break down the bile acid and hydrolyse bile salts ${ }^{(8,11,15)}$. By doing so, the enterohepatic circulation of bile acids will be disrupted $^{(38-40)}$. Hydroxy methyl glutarate CoA (HMG $\mathrm{CoA}$ ) is another compound that helps probiotics block HMG CoA reductase activity, which is a rate-limiting enzyme and is involved in the endogenous production of cholesterol. Probiotic bacteria reduce the absorption of cholesterol in the intestine by binding and hence incorporating it into the cell membrane. Cholesterol can also be assimilated during growth. For the mentioned mechanisms to operate, lactic acid bacteria must remain alive when passing through the gastrointestinal tract. This seemed to be true only in the probiotic yoghurt group, but not in the conventional yoghurt group. All of the above-mentioned activities help with the cholesterol-lowering actions of yoghurt.

In the present study, changes in lipid profile were observed in both probiotic and conventional yoghurt-consuming groups compared with the control group. We did not observe significant differences between lipid profile parameters in the two yoghurt groups. The fact that changes in lipid profile were significant in the yoghurt-consuming groups compared with the control group, and not with each other, leads us to draw the conclusion that any effect is due to the consumption of fermented milks and not necessarily the probiotic yoghurt. The present study showed that both products had a positive effect on the lipid profile of the subjects.

\section{Acknowledgements}

The present study was supported by a grant (no. 6242) from the Vice-chancellor for Research, TUMS, Iran. We are grateful to the Research and Development Division of Iran Dairy Industry Corporation (IDIC-Pegah), in Tehran, that provided the dairy products for the present study.

I. E. initiated the study, supervised the project, and assisted in interpretation and in writing the manuscript. H. S.-Y. conducted the study, carried out statistical analysis and interpretation and wrote the manuscript. A. D. supervised the study and assisted in writing the manuscript. M. J. supervised the laboratory analysis. $\mathrm{R} \mathrm{H}$. supervised the statistical analysis. M. C. assisted in conducting the study.

The authors declare that there are no conflicts of interest.

\section{References}

1. Stamler J \& Neaton JD (2008) The multiple risk factor intervention trial (MRFIT): importance then and now. JAMA 300, $1343-1345$.

2. Imamura T, Doi Y, Arima H, et al. (2009) LDL cholesterol and the development of stroke subtypes and coronary heart disease in a general Japanese population: The Hisayama study. Stroke 40, 350-354.

3. Drisko JA, Giles CK \& Bischoff BJ (2003) Probiotics in health maintenance and disease prevention. Altern Med Rev 8, $143-155$. 
4. Goldin BR \& Gorbach SL (2008) Clinical indications for probiotics: an overview. Clin Infect Dis 46, Suppl. 2, S96-S100.

5. Rautava S \& Isolauri E (2003) Gut microbiota and the intestinal immune system in food allergy - targets for probiotic therapy. Food Allergy Intolerance 4, 5-31.

6. Shi HN \& Walker A (2004) Bacterial colonization and the development of intestinal defences. Can J Gastroenterol 18, 493-500.

7. Mann GV \& Spoerry A (1974) Studies of a surfactant and cholesterolemia in the Maasai. Am J Clin Nutr 27, 464-469.

8. Gilliland SE, Nelson CR \& Maxwell C (1985) Assimilation of cholesterol by Lactobacillus acidophilus. Appl Environ Microbiol 49, 377-381.

9. Lin MY \& Chang FJ (2000) Antioxidative effect of intestinal bacteria Bifidobacterium longum ATCC 15708 and Lactobacillus acidophilus ATCC 4356. Digest Dis Sci 45, 1617-1622.

10. Tahri K, Grill JP \& Schneider F (1996) Bifidobacteria strain behavior toward cholesterol: coprecipitation with bile salts and assimilation. Curr Microbiol 33, 187-193.

11. Klaver FA \& Van Der Meer R (1993) The assumed assimilation of cholesterol by lactobacilli and Bifidobacterium bifidum is due to their bile salt-deconjugating activity. App Environ Microbiol 59, $1120-1124$.

12. Pereira DI \& Gibson GR (2002) Effects of consumption of probiotics and prebiotics on serum lipid levels in humans. Biochem Mol Biol 37, 259-281.

13. Fabian E \& Elmadfa I (2007) The effect of daily consumption of probiotic and conventional yoghurt on oxidant and antioxidant parameters in plasma of young healthy women. Int $J$ Vitam Nutr Res 77, 79-88.

14. Kiessling G, Schneider J \& Jahreis G (2002) Long-term consumption of fermented dairy products over 6 months increases HDL cholesterol. Eur J Clin Nutr 56, 843-849.

15. Gilliland SE \& Walker DK (1989) Factors to consider when selecting a culture of Lactobacillus acidophilus as a dietary adjunct to produce a hypocholesteremic effect in humans. J Dairy Sci 73, 905-911.

16. Lin SY, Ayres JW, Winkler W, et al. (1989) Lactobacillus effects on cholesterol: in vitro and in vivo results. J Dairy Res 72, 2885-2889.

17. Anderson JW \& Gilliland SE (1999) Effect of fermented milk (yogurt) containing Lactobacillus acidophilus L1 on serum cholesterol in hypercholesterolemic humans. J Am Coll Nutr 18, $43-50$.

18. Xiao JZ, Kondo S, Takahashi N, et al. (2003) Effects of milk products fermented by Bifidobacterium longum on blood lipids in rats and healthy adult male volunteers. J Dairy Sci 86, $2452-2461$.

19. Hilvak P, Odraska J, Ferencik M, et al. (2005) One-year application of probiotic strain Enterococcus faecium M-74 decreases serum cholesterol levels. Bratisl Lek Listy 106, 56-72.

20. Greany KA, Bonorden MJL, Hamilton-Reeves JM, et al. (2008) Probiotic capsules do not lower plasma lipids in young women and men. Eur J Clin Nutr 62, 232-237.

21. Ghafarpour M, Houshiar-rad A \& Kianfar H (1999) The Manual for Household Measures Cooking Yield Factors and Edible Portion of Foods. Tehran: Nashre Olume Keshavarzy.

22. Artiss JD \& Zak B (1997) Measurement of cholesterol concentration. In Laboratory Measurement of Lipids Lipoproteins and Apolipoproteins, 3rd ed., pp. 99-114 [N Rifai and GR Warnick, editors]. Washington, DC: AACC Press.
23. Cole TG, Klotzsch SG \& McNamara J (1997) Measurement of triglyceride concentration. In Handbook of Lipoprotein Testing, pp. 115-126 [N Rifai, GR Warnick and MH Dominiczak, editors]. Washington, DC: AACC Press.

24. Rifai N, Bachorik PS \& Alberts JJ (1999) Lipids, lipoproteins, and apolipoproteins. In Tietz Textbook of Clinical Chemistry, 3rd ed., pp. 809-861 [CA Burtis and ER Ashwood, editors]. Philadelphia, PA: Saunders.

25. Hepner G, Fried R, St Jeor S, et al. (1979) Hypocholesterolemic effect of yogurt and milk. Am J Clin Nutr 32, 19-24.

26. Harrison VC \& Peat G (1975) Serum cholesterol and bowel flora in newborn. Am J Clin Nutr 28, 1351-1355.

27. Schaafsma G, Meuling WJA \& Bouley C (1998) Effects of milk product, fermented by Lactobacillus acidophilus and with fructo-oligosaccharides added, on blood lipids in male volunteers. Eur J Clin Nutr 52, 436-440.

28. Agerbaek M, Gerdes LU \& Richelsen B (1995) Hypocholesterolemic effect of a new fermented milk product in healthy middle-aged men. Eur J Clin Nutr 49, 346-352.

29. Richelsen B, Kristensen K \& Pedersen SB (1996) Long-term (6 months) effect of a new fermented milk product on the level of plasma lipoproteins - a placebo-controlled and double blind study. Eur J Clin Nutr 50, 811-813.

30. Imaizumi K, Tominaga A, Sato M, et al. (1992) Effects of dietary sphingolipids on the levels of serum and liver lipids in rats. Nutr Res 12, 543-548.

31. Kobayashi T, Shimizugawa T, Osakabe T, et al. (1997) A longterm feeding of sphingolipids affected the levels of plasma cholesterol and hepatic triacylglycerol but not tissue phospholipids and sphingolipids. Nutr Res 17, 111-114.

32. Vesper H, Schmelz EM, Nikolova-Karakashian MN, et al. (1999) Sphingolipids in food and the emerging importance of sphingolipids to nutrition. J Nutr 129, 1239-1250.

33. Smedman AE, Gustafsson IB, Berglund LG, et al. (1999) Pentadecanoic acid in serum as a marker for intake of milk fat and metabolic risk factors. Am J Clin Nutr 69, 22-29.

34. Samuelson G, Bratteby LE, Mohsen R, et al. (2001) Dietary fat intake in healthy adolescents: inverse relationships between the estimated intake of saturated fatty acids and serum cholesterol. Br J Nutr 85, 333-341.

35. Mensink RP, Zock PL, Kester AD, et al. (2003) Effects of dietary fatty acids and carbohydrates on the ratio of serum total to HDL cholesterol and on serum lipids and apolipoproteins: a meta-analysis of 60 controlled trials. Am J Clin Nutr 77, 1146-1155.

36. Temme EHM, Mensink RP \& Hornstra G (1996) Comparison of the effects of diets enriched in lauric, palmitic or oliec acids on serum lipids and lipoproteins in healthy men and women. Am J Clin Nutr 63, 897-903.

37. Lewis SJ \& Burmeister S (2005) A double-blind placebocontrolled study of the effects of Lactobacillus acidophilus on plasma lipids. Eur J Clin Nutr 59, 776-780.

38. De Boever P \& Verstraete W (1999) Bile salt deconjugation by Lactobacillus plantarum 80 and its implication for bacterial toxicity. J Appl Microbiol 87, 345-352.

39. Ahn YT, Kim GB, Lim KS, et al. (2003) Deconjugation of bile salts by Lactobacillus acidophilus isolates. Int Dairy $J \mathbf{1 3}$, 303-311.

40. Doncheva NI, Antov GP, Softova EB, et al. (2002) Experimental and clinical study on the hypolipidemic and antisclerotic effect of Lactobacillus bulgaricus strain GB N 1 (48). Nutr Res 22, 393-403. 